\title{
I-GALFA: The Inner-Galaxy ALFA Low-Latitude H I Survey
}

\author{
Bon-Chul Koo ${ }^{1}$, Steven J. Gibson ${ }^{2,3}$, Ji-hyun Kang ${ }^{1,2}$, \\ Kevin A. Douglas ${ }^{4,5}$, Geumsook Park ${ }^{1}$, Joshua E. G. Peek ${ }^{5}$, \\ Eric J. Korpela ${ }^{5}$, Carl E. Heiles ${ }^{5}$, and Thomas M. Bania ${ }^{6}$ \\ ${ }^{1}$ Seoul National Univ., KOREA; ${ }^{2}$ Arecibo Obs., USA; ${ }^{3}$ Western Kentucky Univ., USA; \\ ${ }^{4}$ Univ. of Exeter, UK; ${ }^{5}$ Univ. of California - Berkeley, USA; ${ }^{6}$ Boston Univ., USA
}

\begin{abstract}
The I-GALFA survey is mapping HI $21 \mathrm{~cm}$ emission in the inner parts of our Milky Way Galaxy using the Arecibo L-band Feed Array (ALFA). Examples of various H I features such as supershells and chimneys are shown.
\end{abstract}

The I-GALFA survey is mapping all the $\mathrm{H}$ I in the inner Galactic disk visible to the Arecibo $305 \mathrm{~m}$ telescope within 10 degrees of the Galactic plane $\left(\ell=32^{\circ}<l<77^{\circ}\right.$ at $\left.b=0^{\circ}\right)$. The survey, which will obtain $\sim 1.3 \times 10^{6}$ independent spectra, uses the 7-beam Arecibo L-Band Feed Array (ALFA) receiver and will be completed in September 2009. The survey data have a resolution of 3!4, an RMS noise of $\sim 0.25 \mathrm{~K}$ in $0.184 \mathrm{~km} \mathrm{~s}^{-1}$ channels covering LSR velocities of -750 to $+750 \mathrm{~km} \mathrm{~s}^{-1}$. Details of the observing and data reduction can be found in Peek \& Heiles (2008). The data will be made publicly available when the calibrated and gridded cubes are completed. Further information on the I-GALFA project may be found at www.naic.edu/ igalfa.

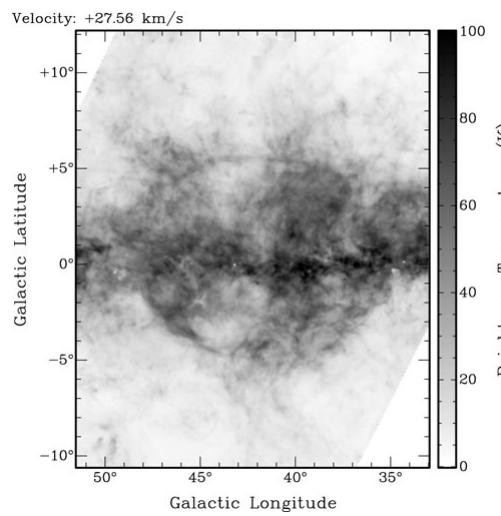

Figure 1: Partial I-GALFA H I line channel maps; more data are being added.

Left: Supershell in the Sagittarius spiral arm.

Right: Disk-halo clouds, chimneys, and worms.

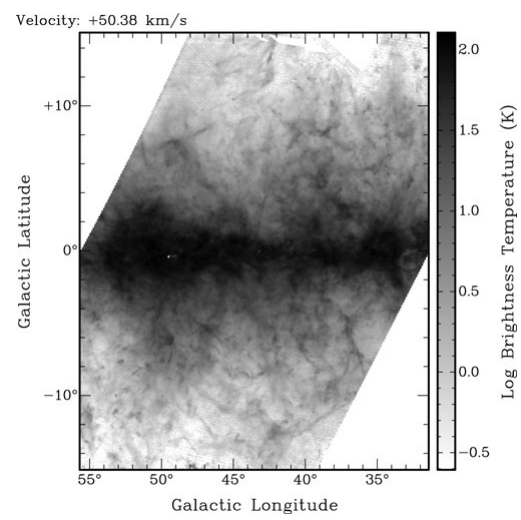

\section{Acknowledgements}

We thank all members of the AO staff for the support of the I-GALFA survey. B.-C. K. is supported by the Korean Research Foundation under grant KRF-2008-313-0409. K. D. was supported by a Marie Curie fellowship. The Arecibo Observatory is part of the National Astronomy and Ionosphere Center, which is operated by Cornell University under a cooperative agreement with the U.S. National Science Foundation.

\section{References}

Peek, J. E. G. \& Heiles, C. 2008, astro-ph arXiv:0810.1283v1 